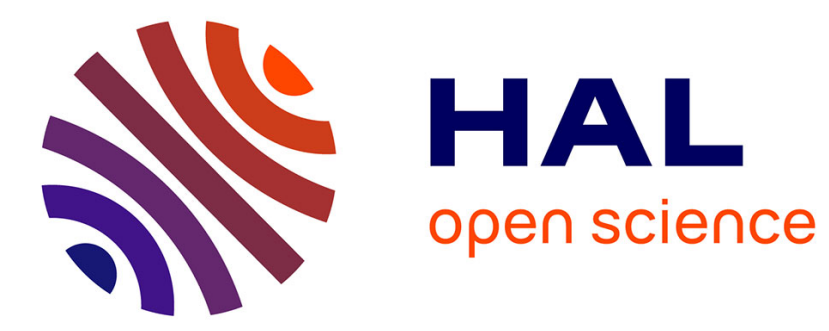

\title{
Contributions of transient absorption and population lens in thermal lens spectroscopy
}

\author{
M. Terazima
}

\section{To cite this version:}

M. Terazima. Contributions of transient absorption and population lens in thermal lens spectroscopy. Journal de Physique IV Proceedings, 1994, 04 (C7), pp.C7-345-C7-352. 10.1051/jp4:1994781 . jpa00253311

\section{HAL Id: jpa-00253311 https://hal.science/jpa-00253311}

Submitted on 1 Jan 1994

HAL is a multi-disciplinary open access archive for the deposit and dissemination of scientific research documents, whether they are published or not. The documents may come from teaching and research institutions in France or abroad, or from public or private research centers.
L'archive ouverte pluridisciplinaire HAL, est destinée au dépôt et à la diffusion de documents scientifiques de niveau recherche, publiés ou non, émanant des établissements d'enseignement et de recherche français ou étrangers, des laboratoires publics ou privés. 
JOURNAL DE PHYSIQUE IV

Colloque C7, supplément au Journal de Physique III, Volume 4, juillet 1994

\title{
Contributions of transient absorption and population lens in thermal lens spectroscopy
}

\author{
M. Terazima \\ Department of Chemistry, Faculty of Science, Kyoto University, Kyoto 606, Japan
}

\begin{abstract}
Contaminations of the population lens and transient absorption in the observed thermal lens signal sometimes cause a misinterpretation of the signal. Recently, we have developed several methods to evaluate the relative contributions of these components in the observed thermal lens signal. These methods with recent results are summarizcd, and merits and limitations of each method are discussed.
\end{abstract}

\section{Introduction}

In the thermal lens (TL) method, a variation of probe beam light density causcd by a nonuniformly spatial shaped excitation beam is detected through a pinhole. Usually the ubserved TL signal is assumed to be composed of the thermal effect due to the radiationless transition from the pholocxcitcd state[1]. Since the signal intensity is proportional to the amount of heat as long as the aborc assumption is correct and the experimental conditions are adequate, it has been used as a sensitive and last response thermometer to trace the dynamics of metastable states [2,3] or chemical reactions [4]. From the time profile or the signal intensity, the quantum yield, the energy of the excited states, reaction cnthalpy and so on can be determined.

However, there are expected to be several origins apart from the heating effect that could change the refractive index of the solution after photoexcitation. For example, the different optical properties between the original state and the metastable state or reaction products will vary the refractive index of the solution and it may contribute to the observed TL signal. Also if the metastable statc absorbs the probe light, the absorption (transient absorption (TA)) varies the probe light intensity and the variation will contaminate the observed TL signal. (Hereafter, we use a notation of "TLex signal" as the variation of the probe light intensity detected under a normal TL experiment; namely, the probe light intensiry is detected through the pinhole. The Thex signal may contain other contributions besides the pure TL signal.) Previously we have shown that the refractive index change due to the different optical properties indeed affects the time profile of the TLex signal and we have referred to that refractive index lens as the population lens (PL) [5]. This work clearly shows that it is important to evaluate such possible contaminations (PL and TA) in order to use the TL method as one of the calorimetric methods. Here we 
intend to show several methods to evaluate the PL and TA in the TLex signal and discuss the merits and limitations. We have chosen the lowest excited triplet $\left(T_{1}\right)$ state of several organic compounds as examples of the metastable states for our demonstrations.

The TLex signal was observed by using a dual beam (pump/probe) configuration setup, which has been described elsewhere in detail [5-7].

\section{Contribution of transient absorption}

If the $T_{1}-T_{n}$ absorption of the probe beam occurs from the metastable $T_{1}$ state, it decreases the probe light intensity. Although the origin of the TA signal is obviously different from that of the TL or PL, both of which appear as the variation of the probe bcam density, the change of the probe light intensity is detected through the pinhole as well as the TL signal and it is rather difficult to separate out or evaluate the contribution of TA from the time resolved TL signal quantitatively. One of the reasons of the difficulty comes from the fact that a very small central area in the probe beam is monitored in the TLex experiment, so that the quantitative measurement of the pure TA intensity using only that arca is very difficult, if not impossible. Also the fact that the decay rate constant of the TA should be the same as the rise time constant of the TL signal (vide infra) makes the separation difficult. Naturally, the different origins of the TA and TL may be used to evaluate the TA contribution. For example, if the TLex signal is detected as a change of the beam spot size by using an imaging detector [8], the contribution of TA in the whole probe beam may be calculated from the integrated probe bcam intensily. Then the TA intensity at the central part can be calculated by using the radial profile of the excitation beam. However applicability of this method is limited by the slow time response of the detector. Here we show two methods to evaluate the TA contribution with the pinhole and a simple photodetector system.

\subsection{Coincident focal point method}

One way to measure the pure TA intensity in the TLex signal is achieved by eliminating the TL signal under the TL experimental conditions. This disappearing condition is fulfilled by adjusting the probe beam waist against that of the excitation beam. It is known that the TL effect is sensitive to the location of the probe beam waist with respect to the excitation beam waist [8]. When the focal point of the probe beam coincides with that of the excitation beam, the lens signal (TL and PL) will disappear and only the TA signal is detected. After the measurement of the time profile of TA (UTA(t)) under this condition, the TA contribution can be subtracted from the observed TLex signal with a different focal point position of the probe beam.

This method has been used to show the existence of PL in the time resolved TLex signal after the photoexcitation of $\mathrm{C} 60$ (a member of fullerenes) for the first time [5]. Although this method can be used generally, relatively fine adjustment of the probe and excitation beams is required. Therefore we think that it is not convenient to be used for routine work.

\subsection{The Concave - Convex Lens (CCL) method}

The TA effect and the refractive index lens cffect could be distinguished by using different probe beam optical paths in the two cases; the TA effect does not change the optical path, while the PL 
or TL effects do. In the concaveconvex lens (CCL) method, the probe beam is collimated at the $T L$ region of the sample $[6,10]$ and then the beam is expanded with a concave or with a convex lens before the pinhole-detector system (Fig.1). When the probe beam is cxpanded by the concave lens, the beam intensity through

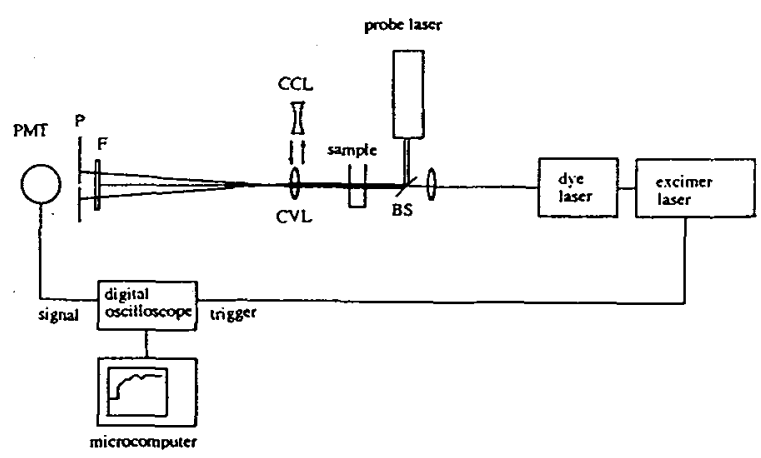
the pinhole should decrease by the TL effect as well as by TA. Fig.1 Experimental setup for the CCL method. Notations; BS; beam On the other hand, when the beam splitter, F; filter, P; pinhole, PMT; photomultiplier. is expanded by the convex lens, the expanded beam due to the TL effect is focused at a farther position from the sample than the unperturbed beam. Then the probe beam intensity through the pinhole increases duc to the TL effect, while the TA contribution decreases the light intensity. By comparing these signals, the contribution of TA can be evaluated.

According to the theory of the ray optics [11], when a lens with a focal length, $I$, is uscd for the expansion, the observed signal $U(t)$ is expressed by [6]

$$
U(t)=A f \Delta n(t)-U T A(t)
$$

where $A$ is a constant and $\Delta n(t)$ is the refractive index variation of the solution duc to the TL and/or PL. Further, the focal length, $f$, is defincd to be negative or positive in the cases of the concave or convex lens, respectively. When one uses the lenses with the same focal length $\left(f_{\mathrm{cc}}=-f_{\mathrm{cv}}\right)$, the simple areragc of two signals measured with the concave $\left(U_{c c}(t)\right)$ and with the convex $\left(U_{c v}(t)\right)$ lenses will give UTA $(t)$.

$$
\mathrm{UTA}(\mathrm{t})=-\left(\mathrm{U}_{\mathrm{cc}}(\mathrm{t})+\mathrm{U}_{\mathrm{cr}}(\mathrm{t})\right) / 2
$$

Applicability of this method has been demonstrated by using samples of quinoxaline and $\mathrm{C} 60$ [6]. The $S_{1}-T_{1}$ intersystem crossing of the two samples takes place very efliciently after the photoexcitation to the excited singlet state. The triplet-triplet (T-T) absorption of quinoxaline at $633 \mathrm{~nm}$ has been known to be weak [12], while the T-T absorption spectrum of $C_{60}$ is located near that wavelength and the absorbance is relatively large [13].

The time dependence of the pure TL signal in the quinoxaline case has been studied. Since the time profile of the pure TL signal reflects the heat releasing process and the decay rate constant from the excited singlet state is much faster than the time resolution of the TL signal, the haat accompanied by the decay of the $S_{1}$ state appears as the fast rise with a time constant limitcd by the instrumental response. On the other hand, decay from the $T_{1}$ state can be slower than the time resolution and the TL signal rise with the same rate constant of the decay process. The total ( $U_{\mathrm{TO} T}^{\mathrm{TL}}$ ) and slow ( $\mathrm{U}_{\mathrm{SLOW}}^{\mathrm{TL}}$ ) rising signal intensities are expressed by [3]

$$
\mathrm{U}_{\mathrm{SLOW}}^{\mathrm{TL}} / \mathrm{U}_{\mathrm{TOT}}^{\mathrm{TL}}=\phi_{\mathrm{isc}}\left(\mathrm{h} v_{\mathrm{ex}}-\phi_{\mathrm{f}} \mathrm{E}_{\mathrm{f}}\right) / \mathrm{ET}_{\mathrm{T}}
$$


where $\phi_{i s c}$ and $\phi_{f}$ are, respectively, the quantum yields of the triplet formation and the fluorescence, ET the energies of the $\mathrm{T}_{1}$ state, $E_{\mathrm{f}}$ average photon energy of the fluorescence, hvex photon energy of the excitation.

The TLex signal after photoexcitation of quinoxaline appears as the decrease of probe light with the concave lens for expansion. The time profile consists of fast and slow rising components, which reflect the heat releasing processes of quinoxaline as explained above. When the convex lens is used for expansion, the TLex signal shows the increase of the probe beam intensity as predicted by eq. ( 1 ) with UTA $\ll$ Af $\Delta n(t)$. The important point is that USLOW/UTOT of the observed signal is essentially the same as in the convex lens case. This fact and eq. (1) indicale that the TA contribution is negligible in the TLex signal, which is consistent with the T-T absorption studies of quinoxaline [12]. On the other hand, there is a difference in the time profiles of the TLex signals after the excitation of $C_{60}$ with the convex lens and with the concave lens. The slow rising component in the signal with the concare lens is much smaller than that with the convex lens, although the time constants of the slow rising signals are similar to each other. The different USLOW/UTOT is due to the contribution of the TA in the carly time region.

From both signals and eq.( 2), UTA in the TLex signal can be reasonably evaluated. These results show that this method is reliable and convenient for many experiments.

\section{Contribution of population lens}

Since the PL signal appears as a result of the probe beam expansion due to the non-uniform distribution of the refractive index in solution, the separation of PL from the pure TL signal is expected to be more difficult. However there are several ways to evaluate the relative contribution in the TLex signal.

\subsection{Probe wavelength dependence}

The refractive index variation due to the TL effect is essentially independent of the probe wavelength, while that due to PL is not. The relationship between the absorption spectrum and the dispersion of the concomitant refractive index change $\left(\Delta \mathrm{n}_{\mathrm{PL}}\right)$ is given by eq. (4) [14] and depicted in Fig. 2 -a .
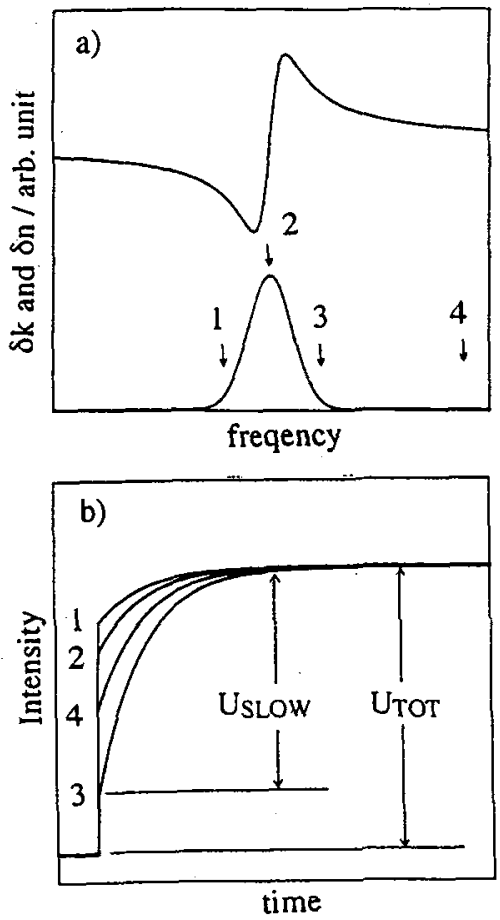

Fig.2 (a) Absorption spectrum (lower curve) and dispersion curve of the concomitant refractive index (upper curve). (b) Expected time resolved Thex signals probed at various frequencies indicated in (a). A time profile of the pure TL signal is curve 4. TA and/or negative $\Delta \mathrm{nPL}_{\mathrm{P}}$ contributions make the slow rising component smaller (curves 1 and 2 ), while positive $\Delta n_{P L}$ makes it larger (curve 3). The intensities of the total (UTOT) and slow rising component (USLOW) are shown in the curve 3 as an example. 


$$
\left.\Delta n_{P L}=\Sigma C_{i}\left(\omega_{0}^{2}-\omega_{i}^{2}\right) /\left\{(\omega r)^{2}-\omega_{i}^{2}\right)^{2}+\gamma i^{2} \omega_{i}^{2}\right\}
$$

where $\omega_{i}$ and $\gamma_{i}$ are the frequency at the absorption maximum and the linewidth of the $i$-th band, respectivcly. If the excited triplet state is the only metastable state as we assume here and the absorption spectra from the ground and the $\mathrm{T}_{1}$ states have been known completely, the contribution of PL could be quantitatively calculated from eq. (4). This expectation is demonstrated as follows.

If the TLex signal is composed of only the pure TL signal, the time profile is expressed by eq. ( 3 ). On the other hand, if PL contaminates the TLex signal, USLOW/UTOT deviates from the expected value from eq. ( 3 ) even after removing the TA contribution. Since the PL contribution should disappear after the excited state has relaxed, UTOT should be composed of the pure TL signal.

$$
U_{S L O W} / U_{T O T}=\left(U_{S L O W}^{\text {TL }}+U_{P L}\right) / U_{T}^{\text {TL }} \text { T }
$$

In this equation, $U_{P L}$ depends on the probe wavelength as predicted by eq. (4), while USLON dose not. Thereforc the time profile, especially USLOW/UTOT, should depend on the probe warelength. Expected time profiles of the TLex signal at various probe wavelengths are shown in Fig.2-b.

We have measured USLOW/UTOT of anthracene in benzene at various probe wavelengths using an Ar ion laser [6] and the values are plotted in Fig.3-a with a reported $T-T$ absorption spectrum [15]. The value decreases with probing at longer wavelength. $U_{P L}$ is obtained from the time dependence of the signal and the literature value $\phi i s c=0.70$ [16]. The UPL/UTLvalues are plotted on Fig.3-b. UPL is also calculated from the reported absorption spectra. The probe wavelength dependence agrees fairly well with the experimental values. This method will give $U_{P L}$ hidden in the TLex signal from the absorption spectra, which are much easier to measure. However, if all absorption bands including far UV region are not known, a possibility of an offset in the calculated $\mathrm{U}_{\mathrm{PL}}$ cannot be excluded completely.

\subsection{Solvent dependence}

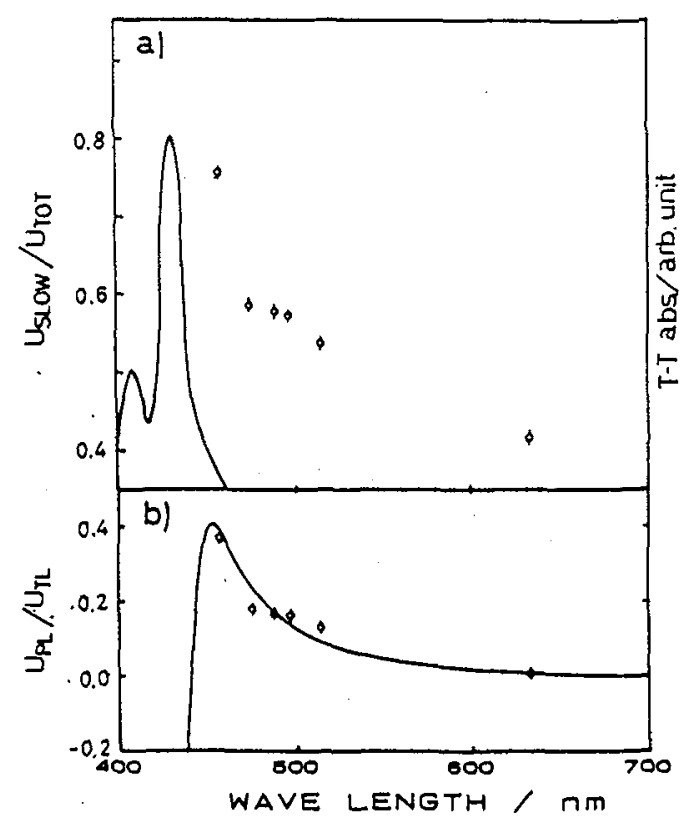

Fig.3 (a) Probe wavelength dependence of USLOW/UTOT in the observed TLex signals of anthracene in benzene and a reported $\mathrm{T}-\mathrm{T}$ absorption spectrum. (b) Relative contribution of PL in the pure TL signal (UPL/UTL) and a theoretically calculated probe wavelength dependence of $U_{P L} / U_{T L}$ from the ground state absorption and the T-T absorption spectra. 
Generally, not all of the absorption spectra from the ground and the $T_{1}$ states of many compounds have been reported. In such cases, the contribution of PL should be determined experimentally not from absorption spectra and eq.(4). Alternatively, we might use a diffcrent effect of thermal properties of the medium to PL and TL. The TL signal intensity depends on the thermal properties of the medium as

$$
\mathrm{UTL}_{\mathrm{TL}}=\mathrm{A}^{\prime} \mathrm{H}(\mathrm{dn} / \mathrm{dT}) / \rho \mathrm{C}_{\mathrm{p}}=\mathrm{A}^{\prime \prime} \mathrm{SC} \text { ex }
$$

where $S$ is defined by $\rho C_{p}$ and $C^{e x}$ is the concentration of the excited state, which should be proportional to the thermal energy, $\mathrm{H}$, from the excited state. Combining eq. ( 3 ) and ( 6 ), one can obtain USLOW/UTOT as

$$
\mathrm{USLOW}_{\mathrm{UTOT}}=\mathrm{U}_{\mathrm{SLOW}}^{\mathrm{TL}} / \mathrm{U}_{\mathrm{TOT}}^{\mathrm{TL}}+\mathrm{D} \Delta \mathrm{n}_{\mathrm{PL}} / \mathrm{Sn}
$$

where $\mathrm{D}$ is a proportionality constant. Since the first term in eq.(7) is determined solely by the photophysical properties of the solute, it is the second term that affects the thermal properties of the medium. Therefore when USLOW/UTOT is measured by changing $S$, the plot of USLOW/UTOT against $1 / \mathrm{Sn}$ should give a straight line with an intercept of USLOW/UTOT. This idea is demonstrated in the case of anthracene by changing the solvents (a series of alkanes [6]). Fig.4 shows the plot of the USLOW/UTOT value probed at $458 \mathrm{~nm}$ against $\mathrm{C} / \mathrm{Sn}$. The contribution of TA is eliminated by the CCL method. Since at this probc wavelength, even a small peak shift of the $\mathrm{T}-\mathrm{T}$ absorption band affects $\Delta \mathrm{n}_{\mathrm{PL}}$, the variation of $\Delta n \mathrm{~nL}_{\mathrm{L}}$ duc to that solvent effect should be taken into consideration by a parameter $\mathrm{C}$, which is calculated

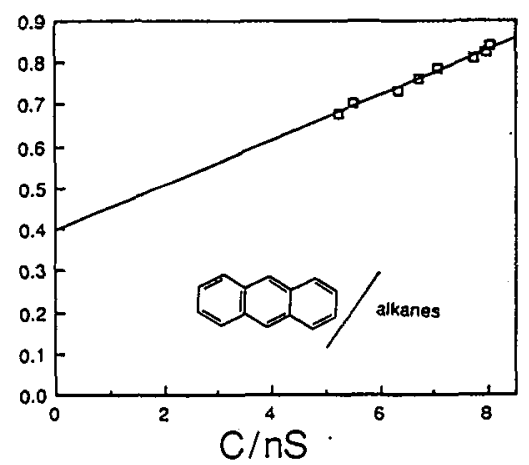

Fig.4 Plot of USLOW/UTOT after the photoexcitation of anthracene in a series of alkanes probed at $458 \mathrm{~nm}$. The straight line is calculated by the least square method. from eq. (4). The plot shows a straight line as expected. The intercept $(0.4)$ gives $\phi_{i s c}=0.70$, which agrees well with the previously determined value. This method should work well when the absorption band is not located near the probe wavelength. When that is the case, a small shift of the absorption band due to the solvent effect will seriously change $\triangle \mathrm{nPL}$ and this method cannot be applied.

\subsection{Rise profile}

In many organic solvents, the origin of the TL signal is the refractive index variation due to the volume expansion of the medium, which is induced by heat. The volume expansion does not take place immediately after the heat release. Therefore there is a finite rise time, which is determinct by the acoustic transit time (around $100 \mathrm{~ns}$ under normal TL experimental conditions). On the other hand, PL is created whenever the metastable state is created and the rise time is determined by the creation rate of the metastable state, which could be an order of 10 ns or less for many compounds. On the basis of 
these facts, it is expected that the PL contribution can be separated from the pure TL signal by analyzing the rise profile of the TLex signal.

Fig.5-a shows the time profile of the TLex signal after the photoexcitation of 9,10diazaphenanthrene (DAP) in benzene. It is already known that DAP possesses very short lived $S_{1}$ and $\mathrm{T}_{1}$ states and the heat releasing rate is considered to be instantaneous. Therefore we can safely neglect the contribution of PL, TA, and the slow heat releasing processes. The TL signal gradually appears after the photoexcitation, it reaches a maximum, then it decreases to take a plateau. The time profilc is very similar to that reported by Bailey et al. in the gas phase with a fast relaxation time [17]. Indeed, we find this time profile can be fitted well by a theoretical calculation from the Navier-Stokes equation and the encrgy transport equation [18]. Fig.5-b depicts the rise profile of the TLex signal of $\mathrm{C}_{60}$ in benzene after subtracting the TA contribution by the CCL method. It is clcar that there is a fust rising component in the

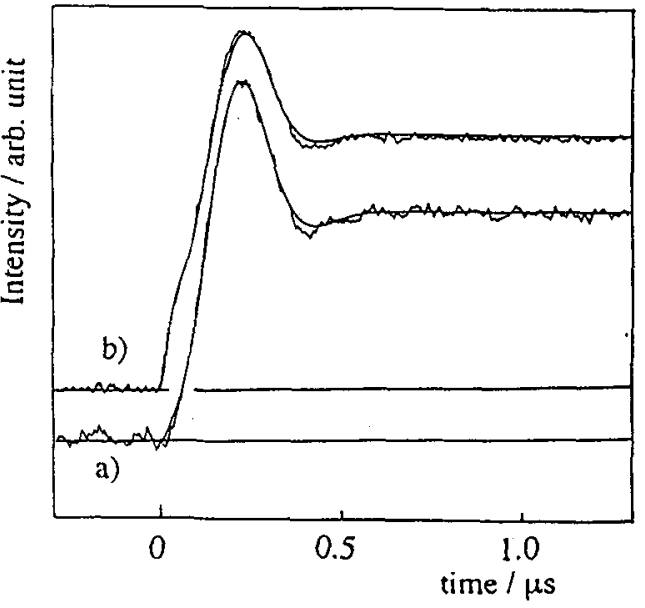

Fig.5 Rise time profiles of the TLex signals af ter the photoexcitation of DAP (a) and C60 (b) in benzene. The smooth curves are best fitted theoretically calculated curves with only the pure TL contribution for (a) and a superposition of the TL and PL contributions for (b). signal. The time profile can be fitted well by the superposition of the TL signal, which has the same time dependence as in the 9,10-diazaphenanthrene case and a single exponential lunction of PL.

$$
\Delta \mathrm{nPL}_{\mathrm{P}}(\mathrm{t})=\Delta \mathrm{n}_{0} \exp (-\mathrm{kt})
$$

The decay rate constant, $k$, represents the decay rate constant of the $\mathrm{T}_{1}$ state of $\mathrm{C}_{60}$. The lit agrecs well with the observed signal and the contribution of PL can be determined quantitatively. The Contribution of PL can be measured from a single cxperiment by using this method and it does not require any information on the absorption spectra.

\section{Conclusion}

For the quantitative application of the TL method to investigate the excited state dynamics and chemical reactions, it is important to ensure that the observed signal is truly a pure TL signal. If the TL signal is contaminated, the time profile as well as the signal intensity no longer reflects the heating effect by the photoexcited molecules or chemical reactions. There are several origins except the TL signal to give a TL like signal, such as the transient absorption, the population lens and do on. Problems are how to detect such an "impure signal" (of course, the impure signal itself could be important for another application) and how to quantitatively evaluate it. In this paper, we present several new methods to evaluate the TA and PL contributions in the observed TLex signal. We have demonstrated the 
applicability of these methods by taking examples of the $T_{1}$ state as the metastable state. Most of them except the rise time profile analysis method can be applicable to the steady state (cw) TL experiment.

\section{Acknowledgment}

The author is indebted to Mr.T.Hara for technical assistance and to Prof.N.Hirota for valuable discussions.

\section{References}

[1] Whinnery J.R., Acc.Chem.Res.,7(1974) 225-231.; Twarowski A.J. and Kliger D.S., Chem.Phys.,20(1977) 253-258,; Fang H.L. and Swofford R.L.,Ultrasensitive Laser Spectroscopies (Kliger D.S. ed. Academic Press New York,1983) pp. 176-232. ; Braslavsky S.E. and Heibel G.E. Chem.Rev.,92(1992) 1381-1410.

[2] Trevor P.L., Rothem T. and Barker J.R., Chem.Phys.68(1982)341-349.;Poston P.E. and Harris J.M.J.Am.Chem.Soc.,112(1990) 644-650. ;Lebedkin S.F. and Klimov A.D., Chem.Phys.Lett.,190 (1992) 313-318.

[3] Terazima M. and Azumi T., Chem.Phys.Lett.,141(1987) 237-240, ibid,145(1988)286-288, ibid, 153(1988) 27-32.

[4] Darec K.,Opt.Comm.,4(1971) 238-;Guckert J.R. and Carr R.W.,J.Phys.Chem.,90(1986)42864294.; Franko M. and Tran C.D.,Rev.Sci.Instrum.,62(1991)2438-2442.

[5] Terazima M. and Hirota N., J.Phys.Chcm.,96( (1992),7147 -7150.

[6] Terazima M., Hara T. and Hirota N., J.Phys.Chem., 97(1993) 10554-10560.

[7] Tcrazima M., Hara T. and Hirota N., J.Phys.Chem., in press.

[8] Miyaishi K., Imasaka T. and Ishibashi N., Anal.Chem.,54(1982)2039-2044. ; Jansen K.L. and Harris J.M., Anal.Chem.,57(1985) 1698-1703.

[9] Berthoud T., Delorme N. and Mauchien P. Anal.Chem.,57(1985) 1216-1219.

[10] Power J.F. and Salin E.D.,Anal.Chem.,60(1988),838-842.;Power J.F., Appl.Opt.,29(1990) 5262.; Shen J., Lowe R.D. and Snook R.D.,Chem.Phys., 165(1992) 385-396.

[11] Yariv A., Qunatum Electronics (3rd ed. John-Wilcy, New York,1989),pp 106-135.

[12] Labhart H., and Heinzelmann W., Organic Molecular Photophysics (Birks J.B. ed. JohnWilcy,1973) pp297-355.

[13] Terazima M., Hirota N., Shinohara Y. and Saito Y., J.Phys.Chem.,95(1991) 9080-9085.

[14] Longhurst R.S., Geometrical and Physical Optics (2nd edition, Wiley, New York (1967).

[15] Land E.J. Proc.Roy.Soc.London,A305(1968)457-471; Bensasson R. and Land E.J., Trans.Farad.Soc.,67(1971) 1904-1915

[16] Medinger T. and Wilkinson F., Trans.Furad.Soc.,6I(1965) 620-630

[17] Bailey R.T., Cruickshank F.R.,Guthrie R.,Pugh D.,and Weir I.J.M.,Mol.Phys.,48(1983)81-95.

[18] Brucck S.R.J., Kildal H. and Belanger L.J., Opt.Commu.,34(1980) 199-204. 